\title{
Improving the Security and Actuation of Wireless Controlled Microvalve
}

\author{
Ajay Chandra Tikka, Said Al-Sarawi, Derek Abbott, Maggie.S.K.Wong and Jordan.D.Schutz \\ The Centre for High Performance Integrated Technologies and Systems (CHiPTec), School of \\ Electrical and Electronic Engineering, University of Adelaide, SA 5005, Australia.
}

\begin{abstract}
A wireless microvalve would have a wide range of applications, including biomedical applications such as fertility control and nano-litre drug delivery. Arguably the most important aspect for such a device is a secure method to actuate the valve, such that it is not actuated through the spectrum of electromagnetic radiation already present in the surrounding environment. Additionally, many of the possible applications are sensitive to electromagnetic (EM) radiation so the device should be designed to only require the minimum amount of EM input to actuate the valve. To overcome this problem, we propose the use of a coded interdigital transducer (IDT) to respond only to a coded signal. For the wireless microvalve to be useful in biomedical applications, the IDT's response to a specifically coded RF signal must be much greater than its response to another coded RF signal, even if the two codes are very similar, i.e. improve the signal ratio of the device. In this research we demonstrate a number of code sequences that have a correlation function such that the peak response is unique and can be used to provide a high signal-to-noise ratio (SNR) surface acoustic wave. That results in a unique activation of the device when the interrogating RF signal code sequence matches the stored code sequence in the device. Also we will investigate the trade-off between the needed code length to ensure secure operation and the area constrain of the device within the context of biomedical application. For this purpose, the IDT is modelled as a pulse compression filter, which correlates the input signal with a stored replica.
\end{abstract}

Keywords: microvalve, SAW, IDT, biomedical applications, coded actuators

\section{INTORDUCTION}

A wireless, battery less microvalve uses no direct electrical power but makes use of the interrogating signal to provide the needed actuation. Such a device can be placed in an inaccessible location thus opening up new host of applications such as flow regulation, on/off switching and sealing of liquids, gases or vacuums [1-3]. For this purpose we utilise an IDT configuration with the SAW device. The IDT transforms this incoming RF signal from the antenna to a SAW and vice versa. Wireless interrogation of the device is possible by connecting the IDT to an antenna. The SAW generated by the IDT propagates along the piezoelectric substrate. The principles of SAW and the SAW generation mechanism in the microvalve are discussed in a previous publication [3].

The high energy density and small size make SAW devices attractive for actuator applications. But on the other hand the device requires a high input power level to drive it, hence it is important to have an efficient antenna design and ensure that the device will only trigger to a uniquely coded RF signal. Both of these objectives can be achieved by modelling the IDT as a pulse compression filter thus combining the narrowband, high Q-factor operation of a band pass filter with a coded reception scheme. The coding gain further improves the Q-factor [4]. Since the wireless microvalve is primarily intended for biomedical applications its important to make the device as small as possible which calls for increase in interrogating frequency, however, the higher the frequency is, the higher is the attenuation of the wave travelling in tissue [2]. As a result there is a trade-off between the penetration depth and device size for a given maximum excitation power. By replacing the conventional wideband pulse excitation with a coded one this trade-off can be alleviated [5].

Smart Structures, Devices, and Systems III, edited by Said F. AI-Sarawi, Proc. of SPIE Vol. 6414, 64140U, (2007) · 0277-786X/07/\$15 - doi: 10.1117/12.696106 
In this paper various coding schemes are analysed and the best coding technique for the application envisaged is outlined. In section 2, we present the interdigital SAW device design, where the structure of the SAW device and its operation as a matched filter are discussed. Section 3 describes the SAW pulse compression filter and the various code sequences that can be used to improve the security and actuation of the microvalve. Section 4 outlines the model construction and the optimization techniques. Section 5 discusses the simulation results and establishes a relation between the code length and strain generated by the microvalve. Conclusion and future work is presented in section 6 .

\section{INTERDIGITAL TRANSDUCER DESIGN}

\subsection{Background information}

The design of an IDT requires an understanding of the piezoelectric effect and particularly the relationship between stress, strain and electric fields within a piezoelectric material. The following is a brief overview of the equations that are involved in the construction of the model presented in section 4.

The relationship between stress $\mathrm{T},\left(\mathrm{N} / \mathrm{m}^{2}\right)$ and strain $\mathrm{S}$, (dimensionless) within a piezoelectric material is defined as [6], [7]:

and

$$
T=F / A
$$

$$
S=\Delta L / L \text {. }
$$

With $F$ being force (N) and $A$ being area $\left(\mathrm{m}^{2}\right)$. Strain $(S)$ is defined as the fractional deformation of the object due to the applied force $F$ ( $\Delta L$ is change in length; $L$ is total length of material). Stress and strain within an object is defined in tensor notation as [6]:

$$
(T)=(c):(S) \text {, }
$$

where $(c)$ is a constant known as the elastic stiffness coefficient or Young's modulus $\left(\mathrm{N} / \mathrm{m}^{2}\right)$. Where $(T)$ and $(S)$ are tensor stress and tensor strain respectively. For current purposes, it is sufficient to relate stress and strain for a simple compressional or shear form, hence the tensor notation can be dropped and Hook's Law can be used ( $c$ is still the elastic stiffness coefficient but in one dimension):

$$
T=c S \text {. }
$$

In a non-piezoelectric material the relationship between electric flux $D$ and the electric field $E$ is [6]:

$$
D=\varepsilon_{r} \varepsilon_{0} E=\varepsilon E,
$$

where $\varepsilon_{r}$ is the relative dielectric permittivity and $\varepsilon_{0}$ is the free space permittivity. Within a piezoelectric material, due to the coupling of electrical and mechanical parameters, the relationship between electric flux $D$ and the electric field $E$ can be written as

$$
D=[e][S]+[\varepsilon] E
$$

where [e] is the piezoelectric constant matrix $\left(\mathrm{C}^{\mathrm{m}} \mathrm{m}^{2}\right),(S)$ is strain and $\mathrm{E}=$ electric field intensity. The permittivity matrix, $[\varepsilon]$ is measured at zero (or constant) strain.

The applied electric field will affect the mechanical characteristics; thus, the relevant equation becomes [5]:

$$
[T]=[c][S]-\left[\mu^{t}\right] E,
$$

where $\left[\mu^{t}\right]$ is the transpose of the piezoelectric constant $[e]$. The above equation shows the relation between the stress $[T]$ 
for a piezoelectric material when a electric field is applied across it causing a strain [S]. Eqn (7) will be utilised in section 4 when we discuss the model construction.

\subsection{IDT construction}

The IDT considered here has the basic structure shown in Fig.1. In this figure $H$ is the width of finger pairs, $D$ is the individual finger width and $W$ is the finger overlap which is also the width of the acoustic beam. The relative polarity of the finger pairs can be determined by simply noting how the fingers are attached to the busbars.

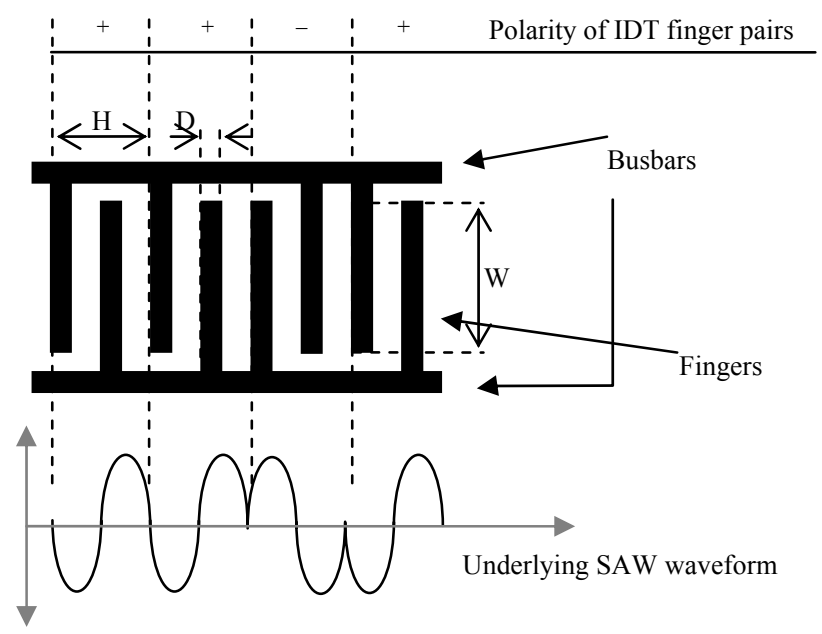

Figure 1: Basic structure of the interdigital transducer showing IDT parameters, relative polarity of finger pairs and the underlying SAW waveform.

For the wireless microvalve design to operate efficiently a full constructive interference of the surface acoustic waves is desired; to achieve this, $H$ must be equal to surface acoustic wavelength $\lambda$ and $D$ must equal $\lambda / 4$ [6], where:

$$
\lambda=v / f
$$

$v$ is the velocity $(\mathrm{m} / \mathrm{s})$ of the acoustic wave and $f$ is the frequency $(\mathrm{Hz})$ of the incoming electromagnetic wave.

\subsection{Matched filter}

The first possible implementation of a secure microvalve is to use a matched filter, which as the name suggests is a filter that is matched to a unique identified signal.

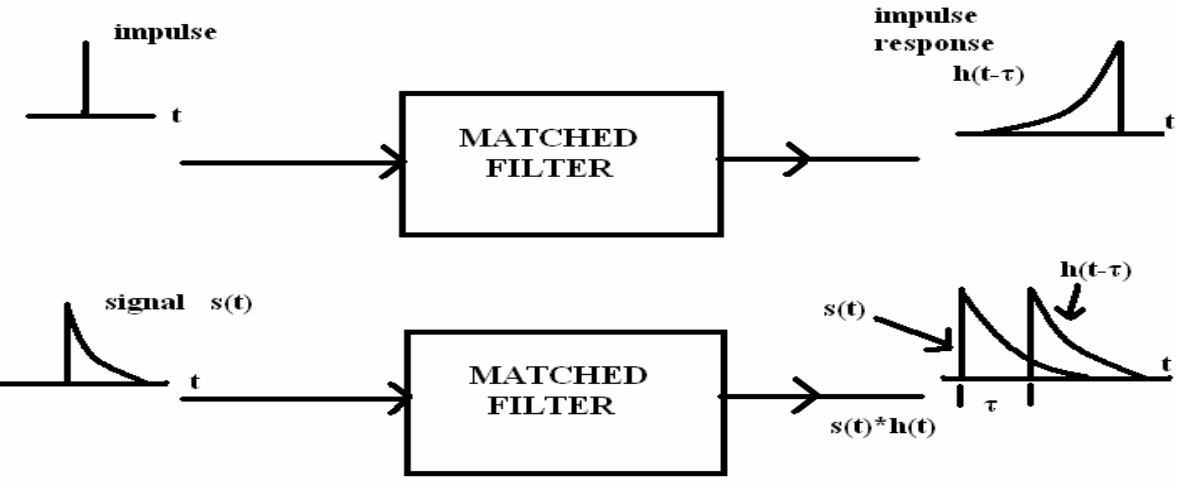

Figure 2: Impulse response of an optimum matched filter [6] 
The signal-to-noise ratio of the signal is maximised if the input signal is 'matched'. The transfer function of the filter is given by:

$$
H(f)=S^{*}(f) e^{-j 2 \pi f T}
$$

Where $T$ is the time when the amplitude of the output signal is maximised and $S^{*}(f)$ is the complex conjugate of the Fourier transform of the input (time-domain) signal $s(t)$.

When the code of the input signal matches the matched filter code the filter produces a high signal to noise ratio. However if another input signal $r(t)$ which is an approximation of the signal $s(t)$, the output of the filter will be at some fraction of the maximum output obtained when the input signal is $s(t)$; the closer $r(t)$ is to $s(t)$, the stronger the output of the filter. Hence a unique activation of the microvalve is not possible. For the wireless microvalve to be effective for biomedical applications, the IDT's response to a coded signal must be much greater than its response to an incorrect signal, even if the incorrect signal is close to the interrogating input signal. Due to this problem with the matched filter, the pulse compression filter method was considered and will be discussed in the following section.

\section{PULSE COMPRESSION FILTER}

A pulse compression filter is also known as a correlation receiver because it correlates the input signal with a stored replica. In our case, the stored replica would be encoded in the IDT. In the frequency domain, compression involves manipulating the phases of the different frequency components of the pulse. Binary phase coding is a way of compressing a pulse where the phase of the radio frequency signal is repeatedly flipped according to the binary code with in the duration of the pulse The compression stage involves correlating the received signal with a replica of the stored code. The length of the transmitted sequence and the efficiency of the compression algorithm determine the output filter signal-to-noise ratio, which is commonly referred to as processing gain.

The appearance of side-lobes around the main signal peak in a pulse compression filter has the adverse effect of interfering with the leak proof operation of the valve. By using complementary codes, which are carefully chosen pairs of codes whose range side-lobes cancel out under ideal conditions, this effect can be minimized. The time-bandwidth product $B T$ determines the effectiveness of a particular code, where $B$ is the pulse bandwidth and $T$ is the pulse duration. The $B T$ for a simple un-coded pulse is 1 , since $B=1 / T$ [9].

The SAW pulse compression filter responds with a correlation peak output only when the interrogator transmits a frequency that matches the microvalve antenna resonance frequency and the signal has the correct code [4]. In order to study the signal processing gain in the SAW device the following terms are defined.

$t=$ time, typically in nsec for $\mathrm{GHz}$ simulations

$f=$ centre frequency of the filter

$N_{B}=$ number of binary bits encoded in the IDT

$T_{B}=\mathrm{N}_{\mathrm{B}} / \mathrm{f}_{\mathrm{c}}=$ bit time of the filter

$f_{B}=1 / \mathrm{T}_{\mathrm{B}}=$ bit rate of the filter

$a_{O}=1,1,1,-1,1, \ldots=$ BPSK input code (e.g. 5-bit Barker sequence).

$b_{o}=1,-1,1,1,1, \ldots=$ time reversed input code encoded in the IDT (e.g. 5-bit Barker sequence).

$u(t)$ is a unit step function

The BPSK coded input signal is given by

$$
V_{i p}(t)=\sin \left(2 \pi f_{c} t\right) \sum_{x=1}^{N_{B}} a_{o}\left[u\left[t-(x-1) \cdot T_{B}\right]-u\left(t-x \cdot T_{B}\right)\right]
$$


The input BPSK signal is modulated by the bit sequence of the similar code encoded in the IDT. The IDT serves as a pulse compression filter to the input BPSK signal. The impulse response of the IDT is given by

$$
C_{I D T}(t)=\sin \left(2 \pi f_{c} t\right) \sum_{x=1}^{N_{B}} b_{o}\left[u\left[t-(x-1) \cdot T_{B}\right]-u\left(t-x \cdot T_{B}\right)\right] .
$$

The electrical input signal, $V_{i p}(t)$ must pass through the IDT to be converted into an acoustic waveform. The convolution operation represents the excitation of the SAW device by the input signal $V_{i p}(t)$, hence the output acoustic wave can be written as

$$
A C_{o p}(t)=\int_{0}^{t} V_{i p}(\tau) \cdot C_{I D T}(t-\tau) d \tau .
$$

When the transmitted code, $a_{O}$ is received by the microvalve, a high signal to noise ratio pulse signal is produced. This pulse is then used to provide the needed activation signal to activate the microvalve.

\subsection{Barker code}

There are sequences known as Barker sequences which are a group of binary codes that have a correlation function such that the peak response is $\mathrm{N}$ times higher than at all other times ( $\mathrm{N}$ is the length of the sequence) [6]. Fig. 3, the correlation of a digital sequence demonstrates the principle idea.

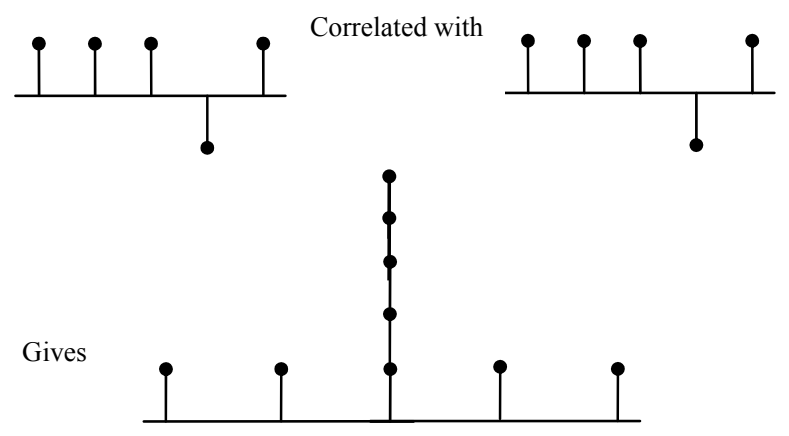

Figure 3: The correlation result of a length 5 Barker sequence. As can be seen, when the signals completely overlap, the result is much greater than at all other points.

True Barker sequences are only known to exist up to a length of 13 [6] (Table 1), however if a longer length is required, multiple sequences can simply be concatenated. For example, a 32-bit code could be constructed using a 13-3-13-3 or an 11-11-7-3 sequence. As the order of the code matters, a 13-13-3-3 code is different to a 3-3-13-13 code, thus allowing many different codes to be constructed for different devices with a significant decrease in the possibility of code reuse.

TABLE.1. KNOWN BARKER SEQUENCES [6]

\begin{tabular}{ll}
\hline \hline & \multicolumn{1}{c}{ SEQUENCE LENGTH, N } \\
\hline 2 & ++ or +- \\
3 & ++- \\
4 & ++-+ or +++- \\
5 & +++-+ \\
7 & +++--+- \\
11 & +++--+-+- \\
13 & +++++--++-++ \\
\hline \hline
\end{tabular}


Now consider the correlation when there is a one bit error in the input signal:

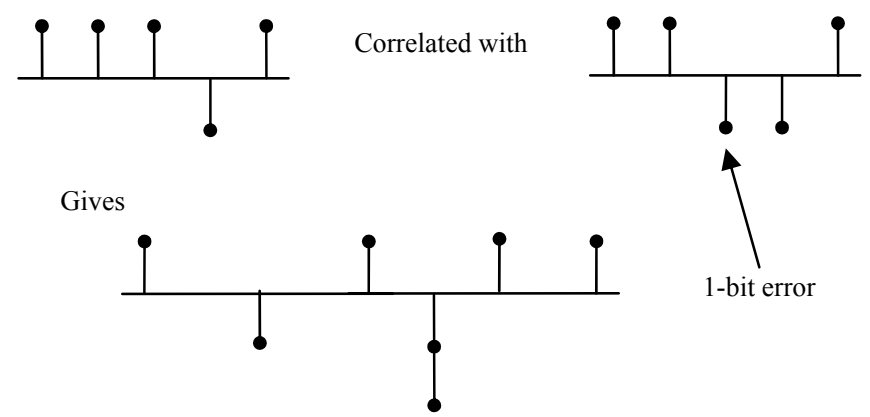

Figure 4: The correlation result of a length 5 Barker sequence, where the input signal has a 1-bit error. The peak magnitude is not as high as in Fig. 3 when the sequences match.

\subsection{Maximum length code}

One more sequence that can be encoded in the IDT is a pseudo-random binary signal called a maximum length sequence (MLS). An MLS signal consists of an apparently random sequence of 0 's and 1's that has a flat frequency spectrum for all frequencies up to the Nyquist frequency with the exception of the dc value [10]. MLS measurements have a very high Signal/Noise ratio. The measurments can be performed in noisy environments as the cross-correlation function reduces the background noise. The $\mathrm{S} / \mathrm{N}$ ratio can be further increased by the use of averaging technique. The peak-tosidelobe ratio of maximal length sequence codes is $(4 \mathrm{~N})^{1 / 2}$. Though this is a high value it is less than the processing gain obtained by using a binary weighted Barker sequence.

\subsection{Golay code}

Golay complementary sequences are pairs of binary codes, where the number of pairs of similar elements with any given separation in first series is equal to the number of unlike elements pairs with the same separation in the second series. It consists of two codes of the same length $\mathrm{N}$ whose auto-correlation functions have side-lobes equal in magnitude but opposite in sign [5]. The peak of the auto-correlation function when both the codes are summed up is $2 \mathrm{~N}$ with no side-lobes.

\begin{tabular}{|c|c|c|}
\hline SEQUENCE LENGTH, N & SEQUENCE & $\begin{array}{l}\text { AMPLITUDE OF } \\
\text { THE MAIN PEAK }\end{array}$ \\
\hline 2 & ++ or +- & 4 \\
\hline 4 & $\begin{array}{l}++-+ \text { or }+++- \\
+++-++-+ \text { or }\end{array}$ & 8 \\
\hline 8 & +++---+- & 16 \\
\hline 16 & $\begin{array}{l}--+----+-+++- \\
+-- \text { or } \\
--+----++--- \\
+-++\end{array}$ & 32 \\
\hline
\end{tabular}

Although these codes may seem to represent the ideal solution to the side lobe suppression problem and thus providing a leak proof operation of the microvalve, they are not appropriate for the current application as the maximum sequence length of the code is limited to 16 . The longest Golay code has a peak-to-sidelobe ratio of 32. After analysing all the codes, the weighted Barker sequence is the ideal for the targeted application as it provides a high processing gain and allows concatenation of basic sequences to form a larger sequence when compared to maximum length code and Golay code. 


\section{MODEL CONSTRUCTION}

\subsection{Model assumptions}

After reviewing the basic principles underpinning the production of a SAW wave using an IDT (and reviewing others attempts and ideas [11] - [15]), the model can now be constructed. Because the calculations of the acoustic wave are numerically and mathematically complex, a number of assumptions were assumed to simplify the device model. These assumptions are:

1) A simple IDT is used, with uniform fingers of $\lambda / 4$ width at $90^{\circ}$ to the busbars with the pairs spaced at $\lambda$ intervals for maximum constructive/destructive interference.

2) The IDT will be directly encoded with Barker sequence(s) (pulse compression filter) to allow actuation of the valve to occur in response to only one particular code.

3) Although the model is representative of all types of surface waves, a Rayleigh SAW propagation mode is used.

The $E$ field can be converted into a stress $[T]$ in the piezoelectric substrate using Eq.7. As there is no external stress, the stress can be written in tensor as:

$$
[T]=[c][S]-\left[\mu^{t}\right] E
$$

Also as we consider the stress in one dimension, longitudinally, the tensor notation in Eq.7 disappears and the above equation can be written as:

$$
T=-\mu_{x x}^{t} E
$$

where $\mu_{x x}^{t}$ is the longitudinal component of the piezoelectric constant mentioned earlier $\left(\mu^{t}\right)$. Although the Rayleigh wave has elliptical motion, the only motion that is being considered here is the component that causes longitudinal stresses.

Because we are utilising the Barker sequence, only the point in the time when all the waves produced by individual finger pairs that result in constructive interference; i.e. the point when the valve should activated is considered. Furthermore, because of the finger spacings, each wave produced by the finger pairs will be completely additive at all points along the substrate. Hence by obtaining the stress produced by a single finger pair, the total stress can be determined by summing up the stress produced by each finger pair. The stress at the valve opening results in the needed electric field between the fingers to cause the actuation.

\subsection{Model equations}

The surface acoustic wave induces an electric potential at the surface of the piezoelectric substrate. A travelling wave of potential V (volts) can be used to model this electric potential [12]

$$
V=V(x, y, t)=a e^{j(\omega t-\beta x)} e^{-\beta|y|},
$$

where $\omega=2 \pi f$ is the angular frequency of the input signal in radians per second, $\beta$ is the wave number in radians per meter, such that $\beta \lambda=2 \pi$ and $\lambda=v / f$ is the acoustic wavelength at SAW velocity $v$. The potential along the propagation direction on the surface is shown by the first term $\mathrm{e}^{\mathrm{j}(\omega t-\beta \mathrm{x})}$. The decaying potential distribution along the axis which is an approximation is specified by the second term $\mathrm{e}^{-\beta|y|}$.

Electric field, $E$ (volts per meter), is generated because of the variation of potential $\mathrm{V}$ on the surface [12]. The electric field in the longitudinal $E_{x}$ and vertical $E_{y}$ direction is given by 


$$
E_{x}=-\frac{\partial V}{\partial x} \quad ; \quad E_{y}=-\frac{\partial V}{\partial y}
$$

As only the longitudinal motion of the SAW is taken into consideration the $E_{y}$ component of the Electric field can be ignored. The total electric field can be written as

$$
E \approx a \beta j e^{-\beta|y|} e^{j(\omega t-\beta x)} .
$$

In a single finger configuration only the peak values are of interest. As a result the $E$ field or stress between the fingers can be approximated as an impulse occurring at the centre of the pair with a value equal to the maximum $E$ field produced between the fingers, $E_{\max }$. This field is converted to stress according to Eq.11. As the polymer is an elastic material, the envelope can be described using an exponential $e^{-\alpha x}$, where $\alpha$ is a parameter dependent on the material that the wave is propagating in through.

As the waves travel in both forwards and backwards along the substrate, it is desired that the exponential peaks at one point and then falls away on both sides. The exponential then becomes $e^{-\alpha|x|}$. This function can be multiplied by the maximum stress induced, so that at its peak (i.e. at the middle point between a finger pair) it is equal to the stress induced by the electric field [14]. This peak can be spatially shifted along the $\mathrm{x}$-axis by substituting for the $\mathrm{x}$ variable so that for a single finger pair stress can be written as

$$
T(x)=-\mu_{x x}^{t} E e^{-\alpha|x-p|},
$$

where $p$ is the value of the $x$-axis where the peak is located and $x$ is the point where the function is to be evaluated. Using Eq.18 the total stress, which is the sum of the stress in all the finger pairs, can be written as

$$
T_{\text {total }}(x)=\sum_{i=1}^{N} T_{i}(x)=-\sum_{i=1}^{N} \mu_{x x}^{t} E e^{-\alpha\left|x-p_{i}\right|},
$$

where $N$ is the number of finger pairs and $p_{i}$ is the positions of the pair at position $i$ along the $x$-axis. By substituting the Electric field from eq.17 and using the relation between stress and strain given in eq.4, the total displacement can be written as

$$
S_{\text {total }}=\frac{T_{\text {total }}(x, t)}{c}=-\frac{j a \beta e^{-\beta|y|} \mu^{t} x x}{c} \sum_{i=1}^{N} e^{\left(-\alpha\left|x-p_{i}\right|+j(\omega t-\beta x)\right)}
$$

The total displacement can be converted to relative displacement using eq. 2 by multiplying eq. 20 by the substrate length.

\subsection{Microvalve optimization}

The efficient operation of a microvalve is affected by a few second order effects. The actuation of the device can be improved by nullifying these second order effects.

All excited IDTs that generate surface waves also generate what are known as bulk waves (which travel within the substrate rather than on the surface) to a certain extent. This is a problem as these waves reflect off the surfaces of the polymer, possibly distorting the surface wave in a random way. The production and effect of bulk waves can be minimised by two design characteristics. Firstly, by keeping the substrate thin, then the area available to the bulk waves is lessened hence reducing their effect. Secondly, an absorption material can be placed on the back of the substrate (the opposing side to the IDT surface) to absorb the bulk waves as they reach the bottom of the substrate. 
Diffraction occurs with the IDT in the same way as it occurs with an incident light beam on a narrow slit. This affects the microvalve as the energy in the desired SAW beam is not focused on the valve opening, but is diffracted. The design approach of ensuring that the valve opening is located in the Fresnel region, rather than the Fraunhofer region can eliminate this problem [6].

\section{MATLAB SIMULATIONS}

Equation (20) was implemented in Matlab using the parameters given in Table 3, for Lithium Niobate (Y-cut) [18]:

Table.3. Simulation parameters

\begin{tabular}{ll}
\hline \hline \multicolumn{1}{c}{ PARAMETER } & \multicolumn{1}{c}{ VALUE } \\
\hline Elastic stiffness coefficient, c & $4.9 \times 10^{9} \mathrm{~N} / \mathrm{m}$ \\
$\alpha$ & 0.005 \\
$\varepsilon_{r}$ & 89 \\
$\varepsilon_{0}$ & $8.854 \times 10^{-12}$ \\
Acoustic wavelength, $\lambda$ & $3.8 \mu \mathrm{m}$ \\
$a$ (voltage) & $10 \mathrm{~V}$ \\
Substrate length & $400 \mu \mathrm{m}$ \\
Operating frequency & $1 \mathrm{GHz}$ \\
\hline \hline
\end{tabular}

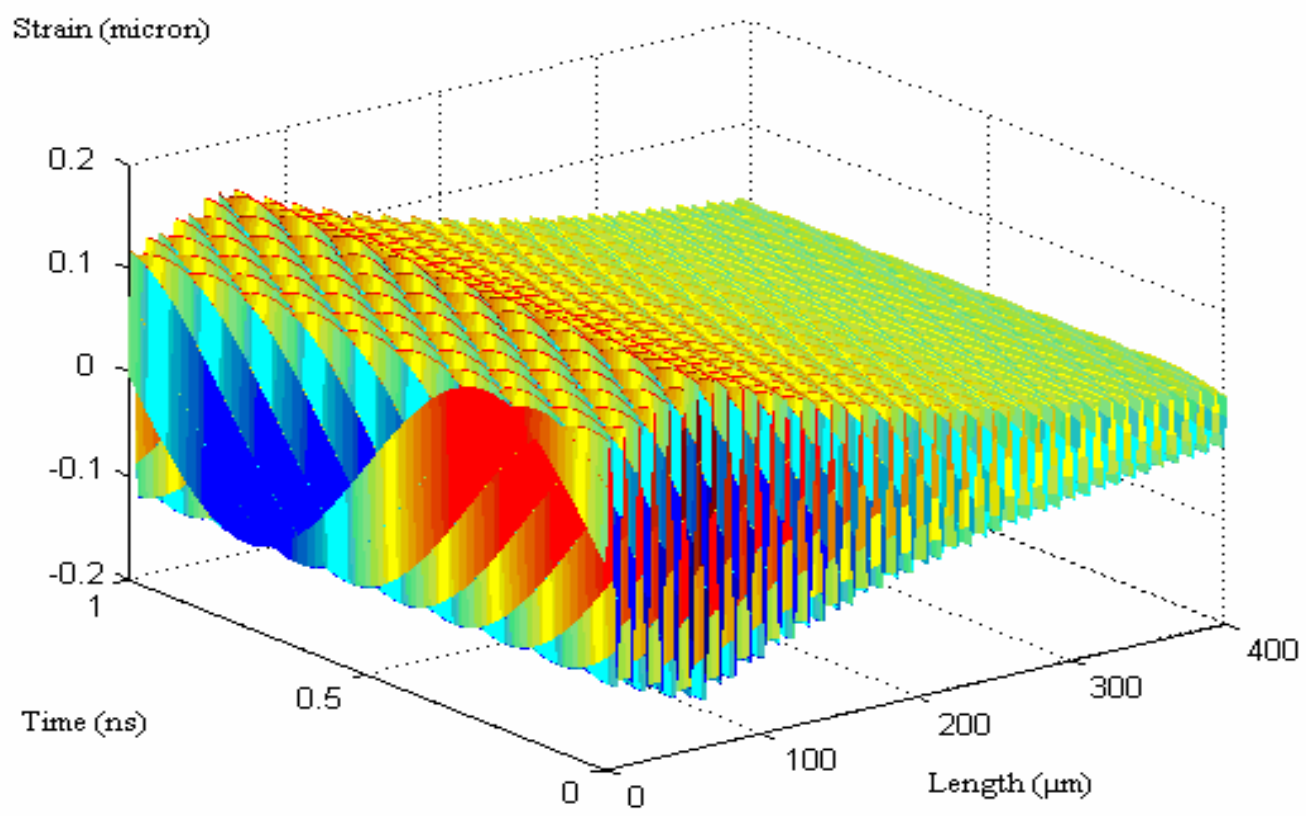

Figure 5(a): Matlab surface plot using 32 IDT fingers 


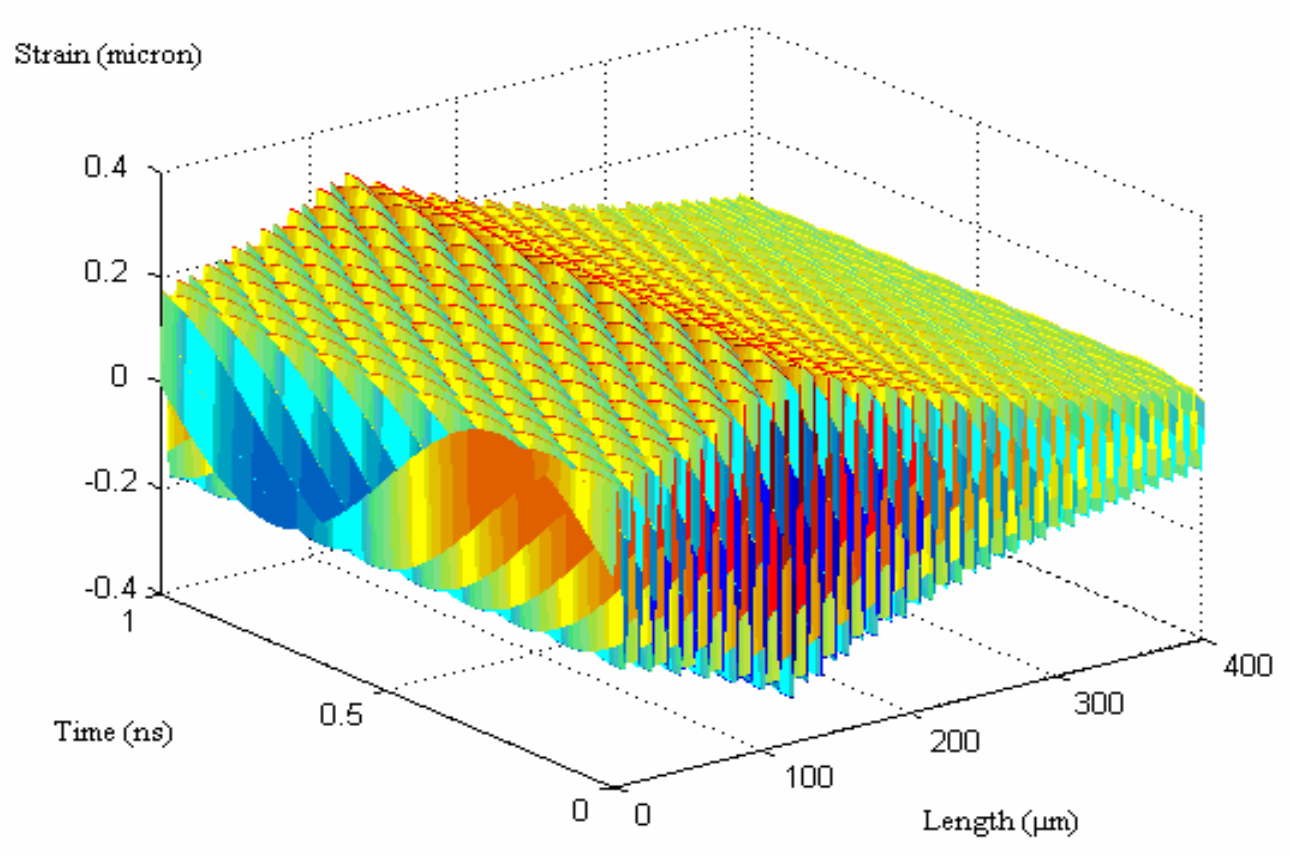

Figure 5(b): Matlab surface plot using 64 IDT fingers

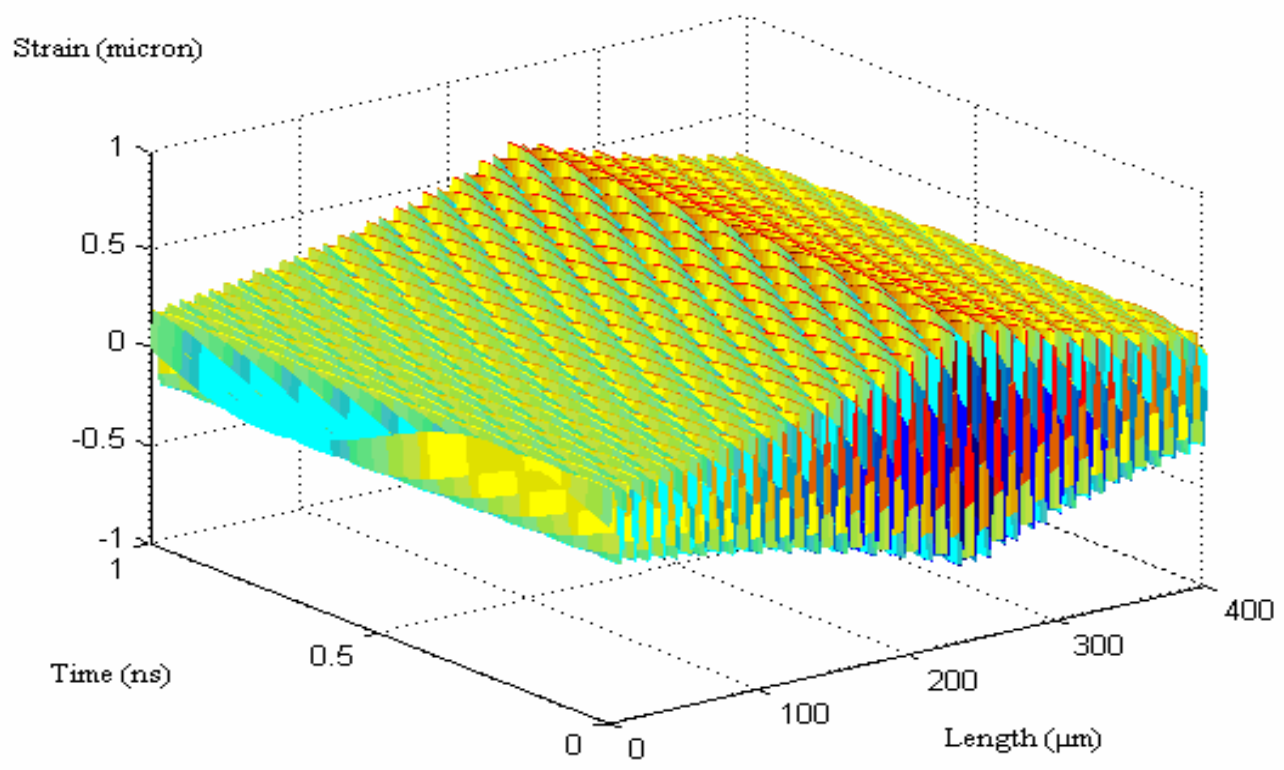

Figure 5(c): Matlab surface plot using 128 IDT fingers

The simulations indicate that as the code length increases the strain increases. So it is desirable to have a longer code length to achieve higher deflection in a microvalve, which can be best attained by using a binary weighted Barker sequence. But there is a trade off between the code length and the length of the SAW device as a bigger microvalve would be ineffective for in-vivo applications. One solution to this problem would be to operate the microvalve at high 
frequencies resulting in reduced finger width enabling a longer code length. From the figures 5(a), 5(b) and 5(c) it can be seen that the maximum strain along the length of the substrate varies with the variations in the code length. For a $32-$ bit, 64-bit and 128-bit coded SAW device the maximum strain was obtained at a substrate length of $120 \mu \mathrm{m}, 160 \mu \mathrm{m}$ and $320 \mu \mathrm{m}$ respectively after the IDT. It was also observed that maximum strain was obtained with a 128-bit coded SAW device. The absolute strain can be calculated by multiplying the relative strain with the length of the substrate, which in turn provides the flexibility of higher deflection in the microvalve. So in order to achieve maximum actuation for the microvalve it is desirable to have the orifice at a point on the piezoelectric substrate where the maximum strain is obtained.

\section{CONCLUSION}

In this paper the ways to improve the security and actuation of a wireless battery-less microvalve were discussed. Such a device being new would open up a wide verity of applications but at the same time its secure operation is of primary interest as a slight malfunction would jeopardise the reliability of the device. The issue is addressed by encoding the SAW devices, where the devices are modelled as a pulse compression filter such that they respond only to a specific coded signal.

The code can be implanted in the IDT by varying the way in which the IDT fingers are connected to the bus-bars. The coding of the IDT not only facilitates a secure actuation of the microvalve but also improves the processing gain of the device. Applicability of the various binary sequences like the Barker sequence, maximum length sequence and Galey sequence to the particular model was investigated. The binary weighted Barker sequence was found to be the appropriate code as it provides a better actuation of the device due to higher processing gain.

After modelling the device it was found that the strain generated by the SAW device increases with the increase in the length of the code. So it is desirable to have as high a code length as possible for high performance microvalve. The next step in research is to simulate the material behavior for SAW devices using this novel technique. The same method can be used to enable multiple access mechanism where multiple devices can be used simultaneously.

\section{ACKNOWLEDGMENT}

The authors would like to thank the Australian Research Council (ARC) and the School of Electrical and Electronics Engineering (University of Adelaide) for the funding and support for the project.

\section{REFERENCES}

[1] K.W.Oh and C.H.Ahn, "A review of microvalves," Journal of Micromechanics and Microengineering, Vol.16, Issue 5, pp. R13-R39, 2006.

[2] L. Ricciardi, I. Pitz, S. Al-Sarawi, V. Varadan and D. Abbott, "Investigation into the Future of RFID in BiomedicalApplications," Proceedings of SPIE Symposium on Bioengineered and Bioinspired Systems, vol. 5119, pp199-209, May 2003.

[3] I. Pitz, L. Hall, H. Hansen, V. Varadan, C. Bertram, S. Maddocks, S. Enderling, D. Saint, S. Al-Sarawi and D.Abbott, "Trade-Offs for Wireless Transcutaneous RF Communication in Biotelemetric Applications," Proceedings of SPIE, vol. 4937, pp 307-318, 2002.

[4] R.W. Brocato, "Passive Microwave Tags," Sandia Report, Sandia National Laboratories, October 2004, Available: http://www.sandia.gov/mstc/documents/saw2.pdf, last accessed: $1^{\text {st }}$ Oct 2006.

[5] A. Nowicki, W. Secomski, I. Trots and J. Litniewski, "Extending penetration depth using coded ultrasonography," Bulletin of the Polish Academy of Sciences Technical Sciences, Vol. 52, No. 3, 2004.

[6] C.K. Campbell, "Surface Acoustic Wave Devices for Mobile and Wireless Communications," chap. 2, 3, 4 and 6, Academic Press: Boston, 1998. 
[7] G.S. Kino, “Acoustic Waves Devices, Imaging \& Analog Signal Processing,” chap. 2, Prentice-Hall, 1987.

[8] Piezoelectric Ceramics: Principles and Applications, APC International Ltd, Available: http://www.americanpiezo.com/piezo_theory/piezo_theory.pdf, last accessed $8^{\text {th }}$ September 2006.

[9] Pulse Compression, the Radar Group, Available: http://www.met.rdg.ac.uk/radar/ufam/pulsecomp.html

[10] J.A.Kemp, "Theoretical and experimental study of wave propagation in brass musical instruments," Phd Thesis, University of Edinburgh, 2002, Available: www.ph.ed.ac.uk/ jonathan/thesis/node82.html, last accessed $10^{\text {th }}$ September 2006.

[11] D. Puccio, D. C. Malocha, D. Gallagher and J. Hines, "SAW Sensors Using Orthogonal Frequency Coding," Proceedings of the 2004 IEEE International Frequency Control Symposium and Exposition, pp 307-310, Aug 2004.

[12] A.V.Mamishev, K.Sundara-Rajan F.Yang, Y.Du and M.Zahn, "Interdigital Sensors and Transducers," Proceedings of the IEEE, vol. 92 no. 5, pp 808-845, May 2004.

[13] Z. C. Feng and C.Chicone, "A delay differential equation model for surface acoustic wave sensors," Sensors and Actuators A: Physical, vol. 104 issue 2, pp 171-178, April 2003.

[14] M.Hofer, N.Finer, S.Zaglmayr, J.Schöberl, G.Kovacs, U.Langer and R.Lerch, "Finite Element Calculation of the Dispersion Relations of Infinitely Extended SAW Structures, Including Bulk Wave Radiation," Proceedings of SPIE, Vol. 4693, p. 472-483, 2002.

[15]Y.Lee, S.Lee and Y.Roh, "Design of Withdrawal-Weighted SAW Filters," IEEE Trans. on Ultrasonics, Ferroelectrics, and Frequency Control, vol. 49 no. 3, pp 337-344, March 2002.

[16] J.W.Gardner, V.K.Varadan and O.O.Awadelkarim, "Microsensors, MEMS, and Smart Devices," chap. 9 and 10, John Wiley \& Sons, 2001.

[17] C.K.Campbell, "Applications of Surface Acoustic and Shallow Bulk Acoustic Wave Devices," Proceedings of the IEEE, vol. 77 no. 10, pp 1453-1484, Oct 1989.

[18] Lithium Niobate- Properties $\mathrm{LiNbO}_{3}$, the Roditi International Corporation Ltd, Available: http://www.roditi.com/SingleCrystal/Lithium_niobate/liNBO3_Properties.html, last accessed $20^{\text {th }}$ Sept 2006. 